B-18-2020

\title{
EL PROBLEMA DE LOS RESIDUOS FLOTANTES EN LA VEGA BAJA DEL SEGURA. UN ANÁLISIS DE LOS ACTORES DEL TERRITORIO
}

\author{
Brugarolas, M.(1)(P), Martínez-Carrasco, L.(2), Rocamora, C.(3), Puerto, H.(4), \\ Abadia, R.(5), Cordero, J.(6)
}

\begin{abstract}
1 Catedrática de Escuela Universitaria, Universidad Miguel Hernández, Dpto. de Economía Agroambiental, Ingeniería Cartográfica y Expresión Gráfica en la Ingeniería, mbrugaro@umh.es

2 Profesora Titular de Universidad, Universidad Miguel Hernández, Dpto. de Economía Agroambiental, Ingeniería Cartográfica y Expresión Gráfica en la Ingeniería, Imartínez@umh.es

3 Profesora Titular de Universidad, Universidad Miguel Hernández, Dpto. de Ingeniería, rocamora@umh.es

4 Profesora Titular de Universidad, Universidad Miguel Hernández, Dpto. de Ingeniería, hpuerto@umh.es

5 Profesor Titular de Universidad, Universidad Miguel Hernández, Dpto. de Ingeniería, abadia@umh.es

6 Profesor de Escuela Universitaria, Universidad Miguel Hernández, Dpto. de Economía Agroambiental, Ingeniería Cartográfica y Expresión Gráfica en la Ingeniería,

jcordero@umh.es
\end{abstract}

\section{Resumen}

En el río Segura y en sus infraestructuras de riego se acumulan residuos sólidos de diversa índole, lo que afecta tanto a la calidad del agua como al propio sistema de transporte de agua por el río ocasionando diversos problemas de difícil solución. Este trabajo tiene como objetivo conocer la visión de los actores del territorio sobre los residuos flotantes en el río Segura y en sus infraestructuras asociadas, concretamente en lo que se refiere a su tipología, sus fuentes y a los problemas que generan. Para ello se realizaron 3 sesiones de grupos focales en las que intervinieron un total de 34 participantes pertenecientes a distintos colectivos, lo que permitía el análisis global desde distintos puntos de vista. Para los participantes, los residuos tienen un origen urbano fundamentalmente, aunque la actividad agrícola y la falta de limpieza de los cauces por parte de la CHS son destacables. En cuanto a los problemas que plantean los residuos, estos son de diversos tipos: económicos, medioambientales y paisajísticos, de salud pública, sociales e incluso legales. El trabajo ha permitido realizar un diagnóstico inicial y es un punto de partida para seguir trabajando en la búsqueda de soluciones.

\section{Abstract}

Solid waste with different origins accumulated in the Segura River and in its irrigation system has important consequences in the water quality and in its transport system. This fact is provoking several problems with a difficult solution. The aim of this work is to know the point of view of the territory actors about this issue, especially its sources and the problems generated. Because of that fact, 3 focus group sessions were carried out with 34 stakeholders from different collectives, therefore a global analysis was obtained. The origin of solid waste was come from urban activities, although agriculture and the lack of cleaning By CHS are highlighted too. Different kinds of problems are originated by solid waste are: economic, 
environmental and landscape, public health social, and legal. The research has allowed to perform an initial diagnosis and it is a good starting point to continue working in the search of solutions. 


\section{1- Introducción}

El recorrido del rio Segura finaliza en la comarca de la Vega Baja del Segura, Ilanura aluvial de unas 23.000 hectáreas, en la que gran parte de su actividad económica gira en torno a su huerta. En dicha huerta se ha desarrollado desde la época romana un sistema de regadío tradicional que comprende un complejo sistema de canales de riego y drenaje que toman el agua directamente desde el río Segura, mediante pequeñas presas o azudes situadas en el lecho del río. Estos azudes, derivan el agua a los canales de riego llamados acequias, que son las encargadas de distribuir el agua a los campos de cultivo. El exceso de agua de riego es recogido en canales de drenaje, llamados azarbes, que a su vez se usan como canales de riego cuando alcanzan la cota suficiente sobre los campos de cultivo (Trapote et al., 2015).

En los últimos años, la infraestructura hidráulica que forma el río Segura a su paso por la Vega Baja, acumula en determinados puntos una gran cantidad de residuos sólidos flotantes, afectando tanto a la calidad del agua como al propio sistema de transporte de agua por el río ocasionando diversos problemas de difícil solución. Los vertidos son de variada procedencia, predominando los residuos vegetales de la ribera del propio río, como las cañas, así como residuos procedentes de la actividad doméstica, agrícola e industrial, entre los que predominan los residuos plásticos. La acumulación de los mismos genera numerosos problemas de tipo económico, paisajístico, medioambiental, de salud pública, social y legal, por lo que se hace necesario analizar estrategias para su reducción o eliminación. Previamente, una acción clave en cualquier estrategia para reducir los residuos es identificarlos y conocer cuáles son sus fuentes (Sandri \& Thompson, 2014).

La economía de la comarca gira en torno a la actividad agroalimentaria y al turismo, actividades en las que el estado del río es determinante. Los actores del territorio directamente relacionados con la gestión del río, como ocurre en otros territorios (Pinto y Maheshwari, 2016; Šebo et al., 2019), están muy sensibilizados, sobre todo en los últimos tiempos en los que las crecidas del río y las inundaciones, consecuencia directa del cambio climático, son cada vez más frecuentes. Es por esto que conocer su visión es el primer paso para proceder a una estrategia general de gestión.

\section{2.- Objetivos}

Este trabajo tiene como objetivo conocer la visión de los actores del territorio sobre los residuos flotantes en el río Segura y en sus infraestructuras asociadas, concretamente en lo que se refiere a su tipología, sus fuentes y a los problemas que generan.

\section{3.- Materiales y métodos}

Se realizaron 3 sesiones de grupos focales en las que intervinieron un total de 34 participantes pertenecientes a distintos colectivos, lo que permitía el análisis global desde distintos puntos de vista. Para la selección de los participantes, se hizo un llamamiento desde la Dirección General del Agua de la Conselleria de Agricultura, Desarrollo Rural, Emergencia Climática y Transición Ecológica. Como participantes, estuvieron representados los siguientes colectivos: Ayuntamientos de la comarca, Confederación Hidrográfica del Segura (CHS), Comunidades de Regantes, asociaciones de agricultores, empresarios, asociaciones ecologistas y voluntarios con vinculación directa con el río. La distribución de los participantes se muestra en la tabla 1.

Tabla 8. Distribución de los participantes en las sesiones focales por colectivo

\begin{tabular}{|l|c|}
\hline Colectivo & $\%$ de asistentes \\
\hline Comunidades de Regantes & 50,0 \\
\hline Empresarios & 14,7
\end{tabular}




\begin{tabular}{|l|l|}
\hline Representantes ayuntamiento & 8,8 \\
\hline Asociación Ecologistas & 8,8 \\
\hline Dirección General del Agua & 5,9 \\
\hline Técnicos responsables de municipios & 5,9 \\
\hline Asociación Agricultores & 2,9 \\
\hline CHS & 2,9 \\
\hline
\end{tabular}

Además, previo al inicio de la sesión, en cada grupo los asistentes cumplimentaron un cuestionario en el que se planteaban diversas cuestiones sobre los tipos de residuos flotantes que se pueden encontrar, sus actividades de procedencia y los problemas que generan los flotantes en el río.

\section{4.- Resultados y discusión}

No se han realizado hasta la fecha demasiados estudios sobre los residuos flotantes en los ríos. Algunos de los más destacados se han realizado en el Danubio (Lechner et al., 2014), en el Támesis (Morritt et al., 2014), en el río Tamar (Sadri and Thompson, 2014), en el río Los Ángeles (Moore et al., 2011) o en el Sena (Dris et al., 2015; Gasperi et al., 2014).

En el caso del Segura a su paso por la Vega Baja, con la información obtenida en las sesiones y de las encuestas se han clasificado los vertidos en diferentes categorías y se han identificado responsabilidades sobre los mismos:

- $\quad$ Cañas y carrizos: la CHS es la responsable de su limpieza y no se está realizando o se realiza de forma insuficiente e inadecuada. Se incide en la necesidad de retirar las cañas una vez cortadas y no dejarlas sobre el talud, ya que finalmente llegan al cauce.

- $\quad$ Basura urbana procedente de la actividad habitual de los habitantes de las poblaciones cercanas y de los mercados semanales: bolsas de plástico, botellas de productos de limpieza personal y del hogar, toallitas que se tiran al inodoro...

- $\quad$ Basura procedente de pequeñas industrias, entre las que destacan los talleres, que no tienen un sistema adecuado de eliminación de residuos sólidos o que no utilizan el existente por disminuir sus costes. En algunos casos, pueden ser actividades no dadas de alta. En este caso, los productos son: neumáticos, componentes industriales, botellas, etc.

- $\quad$ Animales: algunas empresas no llevan al crematorio a los animales muertos y prefieren tirarlos al río.

- Botellas de agua: procedentes de actividades de ocio y deportivas, y también de los trabajadores de la actividad agrícola, aunque en menor medida.

- Restos de bandejas de semilleros, botes de productos químicos: procedentes de la actividad agrícola.

- Bolsas de basura completas, procedentes de viviendas diseminadas a lo largo del cauce.

- Otros residuos vertidos a los imbornales, que algunos usuarios utilizan de papeleras.

La composición de residuos, entre los que predominan la materia orgánica y los plásticos, está en línea con lo encontrado en otros trabajos (Suaria et al, 2015; Rochman et al., 2016; Vlachogianni et al., 2018).

En el cuestionario también se obtuvo información sobre la asignación de responsabilidad en la generación de diferentes residuos flotantes que acaban en el cauce del río: un $56,9 \%$ de los participantes señala que los culpables son los ciudadanos, seguido de un porcentaje importante de encuestados que señalan que los principales responsables son los agricultores $(21,8 \%)$, la confederación hidrográfica es señalada por el $6,9 \%$ de los respondientes, asignándosele la responsabilidad de limpieza del material vegetal que circunda el cauce y que irremediablemente cae en él si no se elimina. Otros colectivos también fueron señalados aunque con una frecuencia mucho menor: excursionistas, ganaderos, industrias o empresas 
de mantenimiento. En cuanto a las principales actividades que provocan mayor generación de residuos, un $46,4 \%$ señala la actividad urbana/doméstica, un $29,1 \%$ menciona la actividad agrícola. En tercer lugar, se menciona el ocio con un $8,7 \%$ de encuestados. En la bibliografía, se encuentran trabajos que relacionan la contaminación por plástico con la abundancia de población (Kwon et al., 2014; Munari, 2016). Baldwin et al. (2016) encuentran una correlación positiva entre núcleos urbanos y negativa con importancia de la actividad agrícola. Otros autores, sí correlacionan las actividades del territorio con la abundancia de residuos (Wan et al., 2018).

Finalmente, se preguntó a los participantes por los problemas que generan los flotantes. Dichos problemas se han agrupado en seis categorías:

1. Económicos: directamente relacionados con la actividad agrícola, como pérdida de valor de los productos agroalimentarios, porque la calidad de las aguas es peor o la dificultad para certificarse en agricultura ecológica; pérdida de valor turístico por la degradación del paisaje y coste de las labores de limpieza y sanciones que tienen que soportar las comunidades de regantes. Los problemas económicos relacionados con las actividades económicas al territorio han sido también analizados en otros trabajos (Jang et al., 2014; Krelling et al., 2017; Williams et al., 2016; Quiang et al., 2020).

2. Medioambientales y paisajísticos: riesgo alto de inundaciones, contaminación de agua del río y del mar, contaminación de parcelas, contaminación de las playas, efecto en la fauna y en la flora, alteración del paisaje o impacto visual que afecta al valor turístico. Otros trabajos destacan la importancia de estos problemas (Allen et al., 2012; Browne et al., 2008; Fossi et al., 2018; Gall y Thompson, 2015; Murray y Cowie, 2011; Rochman et al., 2013; Thompson et al., 2009; Morrit et al., 2014).

3. Salud pública: residuos contaminantes en alimentos y problemas derivados del agua estancada, como los analizados por otros autores (Foekema et al., 2013; Lusher et al, 2013).

4. Sociales: conflictos entre regantes y Confederación, entre ayuntamientos y vecinos y entre usuarios (los de aguas abajo culpan a los de aguas arriba).

5. Legales: trabas burocráticas de la CHS para la retirada de residuos, competencias entre actores no adecuadamente delimitadas.

Sobre los dos últimos tipos de problemas señalados no se han encontrado trabajos que expongan situaciones parecidas.

\section{5.- Conclusiones y recomendaciones}

Analizar, desde un punto de vista de los actores del territorio, las problemáticas a las que se enfrentan, supone conocer de primera mano su opinión, en algunos casos subjetiva. Discutir entre diversos actores procedentes de diversos colectivos, a veces enfrentados, enriquece la visión general del problema.

En el trabajo se ha recogido la visión de los principales implicados en la gestión del río sobre los causantes de los vertidos y las problemáticas que plantea. El análisis de sus opiniones ha permitido realizar un diagnóstico inicial y es un punto de partida para seguir trabajando en la búsqueda de soluciones.

Nuestra propuesta es que en esta búsqueda de soluciones participen, además de los colectivos implicados en la gestión del río, los ciudadanos en general y que se tengan en cuenta métodos participativos como los utilizados en la metodología de este trabajo en lo que a soluciones se refiere, ya que se adaptarán mejor al territorio y a sus integrantes.

\section{8.- Agradecimientos}

Este póster muestra los resultados parciales de la investigación realizada con la financiación de la Dirección General del Agua de la Conselleria de Agricultura, Desarrollo Rural, Emergencia Climática y Transición Ecológica. 


\section{9- Bibliografía}

Allen, R., Jarvis, D., Sayer, S., \& Mills, C. (2012). Entanglement of grey seals Halichoerus grypus at a haul out site in Cornwall, UK. Marine pollution bulletin, 64(12), 2815-2819.

Baldwin, A. K., Corsi, S. R., \& Mason, S. A. (2016). Plastic debris in 29 Great Lakes tributaries: relations to watershed attributes and hydrology. Environmental Science \& Technology, 50(19), 10377-10385.

Browne, M. A., Dissanayake, A., Galloway, T. S., Lowe, D. M., \& Thompson, R. C. (2008). Ingested microscopic plastic translocates to the circulatory system of the mussel, Mytilus edulis (L.). Environmental science \& technology, 42(13), 5026-5031.

Dris, R., Gasperi, J., Rocher, V., Saad, M., Renault, N., \& Tassin, B. (2015). Microplastic contamination in an urban area: a case study in Greater Paris. Environmental Chemistry, 12(5), 592-599.

Kwon, B. G., Saido, K., Koizumi, K., Sato, H., Ogawa, N., Chung, S. Y., ... \& Kogure, K. (2014). Regional distribution of styrene analogues generated from polystyrene degradation along the coastlines of the North-East Pacific Ocean and Hawaii. Environmental pollution, 188, 45-49.

Foekema, E. M., De Gruijter, C., Mergia, M. T., van Franeker, J. A., Murk, A. J., \& Koelmans, A. A. (2013). Plastic in North sea fish. Environmental science \& technology, 47(15), 88188824.

Fossi, M. C., Panti, C., Baini, M., \& Lavers, J. L. (2018). A review of plastic-associated pressures: cetaceans of the Mediterranean Sea and eastern Australian shearwaters as case studies. Frontiers in Marine Science, 5, 173.

Gall, S. C., \& Thompson, R. C. (2015). The impact of debris on marine life. Marine pollution bulletin, 92(1-2), 170-179.

Gasperi, J., Dris, R., Bonin, T., Rocher, V., \& Tassin, B. (2014). Assessment of floating plastic debris in surface water along the Seine River. Environmental pollution, 195, 163-166.

Jang, Y. C., Hong, S., Lee, J., Lee, M. J., \& Shim, W. J. (2014). Estimation of lost tourism revenue in Geoje Island from the 2011 marine debris pollution event in South Korea. Marine Pollution Bulletin, 81(1), 49-54.

Krelling, A. P., Williams, A. T., \& Turra, A. (2017). Differences in perception and reaction of tourist groups to beach marine debris that can influence a loss of tourism revenue in coastal areas. Marine Policy, 85, 87-99.

Lechner, A., Keckeis, H., Lumesberger-Loisl, F., Zens, B., Krusch, R., Tritthart, M., ... \& Schludermann, E. (2014). The Danube so colourful: a potpourri of plastic litter outnumbers fish larvae in Europe's second largest river. Environmental pollution, 188, 177-181.

Lusher, A. L., Mchugh, M., \& Thompson, R. C. (2013). Occurrence of microplastics in the gastrointestinal tract of pelagic and demersal fish from the English Channel. Marine pollution bulletin, 67(1-2), 94-99.

Moore, C. J., Lattin, G. L., \& Zellers, A. F. (2011). Quantity and type of plastic debris flowing from two urban rivers to coastal waters and beaches of Southern California. Revista de Gestão Costeira Integrada-Journal of Integrated Coastal Zone Management, 11(1), 65-73.

Morritt, D., Stefanoudis, P. V., Pearce, D., Crimmen, O. A., \& Clark, P. F. (2014). Plastic in the Thames: a river runs through it. Marine Pollution Bulletin, 78(1-2), 196-200.

Munari, C., Corbau, C., Simeoni, U., \& Mistri, M. (2016). Marine litter on Mediterranean shores: analysis of composition, spatial distribution and sources in north-western Adriatic beaches. Waste management, 49, 483-490.

Murray, F., \& Cowie, P. R. (2011). Plastic contamination in the decapod crustacean Nephrops norvegicus (Linnaeus, 1758). Marine pollution bulletin, 62(6), 1207-1217.

Pinto, U., \& Maheshwari, B. L. (2016). Community perspectives on managing health of periurban river system: evidence from the Hawkesbury-Nepean river catchment, Australia. Journal of Environmental Planning and Management, 59(7), 1257-1276.

Qiang, M., Shen, M., \& Xie, H. (2020). Loss of tourism revenue induced by coastal environmental pollution: a length-of-stay perspective. Journal of Sustainable Tourism, 28(4), $550-567$. 
Rochman, C. M., Hoh, E., Kurobe, T., \& Teh, S. J. (2013). Ingested plastic transfers hazardous chemicals to fish and induces hepatic stress. Scientific reports, 3, 3263.

Šebo, J., Gróf, M., \& Šebová, M. (2019). A contingent valuation study of a polluted urban lake in Košice, Slovakia: The case of the positive distance effect. Journal of environmental management, 243, 331-339.

Suaria, G., Melinte-Dobrinescu, M. C., Ion, G., \& Aliani, S. (2015). First observations on the abundance and composition of floating debris in the North-western Black Sea. Marine environmental research, 107, 45-49.

Thompson, R. C., Moore, C. J., Vom Saal, F. S., \& Swan, S. H. (2009). Plastics, the environment and human health: current consensus and future trends. Philosophical Transactions of the Royal Society B: Biological Sciences, 364(1526), 2153-2166.

Trapote, A., Roca, J.F., Melgarejo, J. (2015): Azudes y acueductos del sistema de riego tradicional de la Vega Baja del Segura (Alicante, España). Investigaciones Geográficas 63, 143-160.

Sadri, S. S., \& Thompson, R. C. (2014). On the quantity and composition of floating plastic debris entering and leaving the Tamar Estuary, Southwest England. Marine pollution bulletin, 81(1), 55-60.

Vlachogianni, T., Fortibuoni, T., Ronchi, F., Zeri, C., Mazziotti, C., Tutman, P., ... \& Mandić, M. (2018). Marine litter on the beaches of the Adriatic and Ionian Seas: An assessment of their abundance, composition and sources. Marine pollution bulletin, 131, 745-756.

Wan, J., Wang, Y., Cheng, M., Engel, B. A., Zhang, W., \& Peng, H. (2018). Assessment of debris inputs from land into the river in the Three Gorges Reservoir Area, China. Environmental Science and Pollution Research, 25(6), 5539-5549.

Williams, A. T., Rangel-Buitrago, N. G., Anfuso, G., Cervantes, O., \& Botero, C. M. (2016). Litter impacts on scenery and tourism on the Colombian north Caribbean coast. Tourism Management, 55, 209-224. 